\title{
696.
}

\section{CALCULATION OF THE MINIMUM N.G.F. OF THE BINARY SEVENTHIC.}

[From the American Journal of Mathematics, t. II. (1879), pp. 71-84.]

For the binary seventhic $(a, \ldots \nmid x, y)^{7}$ the number of the asyzygetic covariants $(a, \ldots)^{\theta}(x, y)^{\mu}$, or say of the deg-order $(\theta, \mu)$, is given as the coefficient of $a^{\theta} x^{\mu}$ in the function

$$
\frac{1-x^{-2}}{1-a x^{7} \cdot 1-a x^{5} \cdot 1-a x^{3} \cdot 1-a x \cdot 1-a x^{-1} \cdot 1-a x^{-3} \cdot 1-a x^{-5} \cdot 1-a x^{-7}}
$$

developed in ascending powers of a. See my "Ninth Memoir on Quantics," Phil. Trans., t. CLXI. (1871), pp. 17-50, [462].

This function is in fact.

$$
=A(x)-\frac{1}{x^{2}} A\left(\frac{1}{x}\right)
$$

where, developing in ascending powers of $a$, the second term $=\frac{1}{x^{2}} A\left(\frac{1}{x}\right)$ contains only negative powers of $x$, and it may consequently be disregarded: the number of asyzygetic covariants of the deg-order $(\theta, \mu)$ is thus equal to the coefficient of $a^{\theta} x^{\mu}$ in the function $A(x)$, which function is for this reason called the Numerical Generating Function (N.G.F.) of the binary seventhic; and the function $A(x)$ expressed as a fraction in its least terms is said to be the minimum N.G.F.

According to a theorem of Professor Sylvester's (Proc. Royal Soc., t. xxvIII. (1878), pp. 11-13), this minimum N.G.F. is of the form

$$
\frac{Z_{0}+a Z_{1}+a^{2} Z_{2}+\ldots+a^{36} Z_{36}}{1-a x .1-a x^{3} .1-a x^{5} .1-a x^{7} \cdot 1-a^{4} .1-a^{6} \cdot 1-a^{8} \cdot 1-a^{10} \cdot 1-a^{12}}
$$


where $Z_{0}, Z_{1}, \ldots, Z_{36}$ are rational and integral functions of $x$ of degrees not exceeding 14 ; and where, as will presently be seen, there is a symmetry in regard to the terms $Z_{0}, Z_{36} ; Z_{1}, Z_{35}$; \&c., equidistant from the middle term $Z_{18}$, such that the terms $Z_{0}, \ldots, Z_{18}$ being known, the remaining terms $Z_{19}, \ldots, Z_{36}$ can be at once written down.

Using only the foregoing properties, I obtained for the N.G.F. an expression which I communicated to Professor Sylvester, and which is published, Comptes Rendus, t. LXXXVII. (1878), p. 505, but with an erroneous value for the coefficient of $a^{7}$ and for that of the corresponding term $a^{29}$.* The correct value is

Numerator of Minimum N.G.F. is =

$$
\begin{aligned}
& 1 \\
& +a\left(-x-x^{3}-x^{5}\right) \\
& +a^{2}\left(x^{2}+x^{4}+2 x^{6}+x^{8}+x^{10}\right) \\
& +a^{3}\left(-x^{7}-x^{9}-x^{11}-x^{13}\right) \\
& +a^{4}\left(2 x^{4}+x^{8}+x^{14}\right) \\
& +a^{5}\left(x+2 x^{3}-x^{9}-x^{11}\right) \\
& +a^{6}\left(-1+2 x^{2}-x^{4}-x^{8}-x^{10}+x^{12}\right) \\
& +a^{7}\left(4 x+x^{3}+3 x^{5}-x^{9}+x^{11}\right) \\
& +a^{8}\left(2-x^{2}-3 x^{6}-3 x^{8}-x^{10}-x^{12}\right) \\
& +a^{9}\left(x+3 x^{3}+x^{5}-x^{7}+2 x^{9}+2 x^{13}\right) \\
& +a^{10}\left(-1+4 x^{2}-x^{6}-2 x^{8}-2 x^{10}-x^{14}\right) \\
& +a^{11}\left(5 x+3 x^{3}+2 x^{5}-x^{7}-2 x^{9}-x^{11}+x^{13}\right) \\
& +a^{12}\left(5+x^{2}-4 x^{6}-6 x^{8}-4 x^{10}-x^{12}+2 x^{14}\right) \\
& +a^{13}\left(x-4 x^{5}-4 x^{7}-x^{9}+x^{11}+4 x^{13}\right) \\
& +a^{14}\left(2+5 x^{2}+x^{4}+x^{6}-2 x^{8}+3 x^{12}-x^{14}\right) \\
& +a^{15}\left(3 x-x^{3}-x^{5}-7 x^{7}-5 x^{9}-x^{11}-x^{13}\right) \\
& +a^{16}\left(6+3 x^{2}+3 x^{4}-4 x^{6}-3 x^{8}-x^{12}+5 x^{14}\right) \\
& +a^{17}\left(-x-2 x^{3}-9 x^{5}-8 x^{7}-4 x^{9}-3 x^{11}+4 x^{13}\right) \\
& +a^{18}\left(2+6 x^{2}+x^{4}+2 x^{6}+2 x^{8}+x^{10}+6 x^{12}+2 x^{14}\right) \\
& +a^{19}\left(4 x-3 x^{3}-4 x^{5}-8 x^{7}-9 x^{9}-2 x^{11}-x^{13}\right) \\
& +a^{20}\left(5-x^{2}-3 x^{6}-4 x^{8}+3 x^{10}+3 x^{12}+6 x^{14}\right) \\
& +a^{21}\left(-x-x^{3}-5 x^{5}-7 x^{7}-x^{9}-x^{11}+3 x^{13}\right) \\
& +a^{22}\left(-1+3 x^{2}-2 x^{4}+x^{8}+x^{10}+5 x^{12}+2 x^{14}\right) \\
& +a^{23}\left(4 x+x^{3}-x^{5}-4 x^{7}-4 x^{9}+x^{13}\right) \\
& +
\end{aligned}
$$

* The existence of these errors was pointed out to me by Professor Sylvester in a letter dated 13th November, 1878.

C. $\mathrm{x}$. 


$$
\begin{aligned}
& +a^{24}\left(2-x^{2}-4 x^{4}-6 x^{6}-4 x^{8}+x^{10}+5 x^{14}\right) \\
& +a^{25}\left(x-x^{3}-2 x^{5}-x^{7}+2 x^{9}+3 x^{11}+5 x^{13}\right) \\
& +a^{26}\left(-1-2 x^{4}-2 x^{6}-x^{8}+4 x^{10}-x^{14}\right) \\
& +a^{27}\left(2 x+2 x^{5}-x^{7}+x^{9}+3 x^{11}+x^{13}\right) \\
& +a^{28}\left(-x^{2}-x^{4}-3 x^{6}-3 x^{8}-x^{12}+2 x^{14}\right) \\
& +a^{29}\left(x^{3}-x^{5}+3 x^{9}+x^{11}+4 x^{13}\right) \\
& +a^{30}\left(x^{2}-x^{4}-x^{6}-x^{10}+2 x^{12}-x^{14}\right) \\
& +a^{31}\left(-x^{3}-x^{5}+2 x^{11}+x^{13}\right) \\
& +a^{32}\left(1+x^{6}+2 x^{10}\right) \\
& +a^{33}\left(-x-x^{3}-x^{5}-x^{7}\right) \\
& +a^{34}\left(x^{4}+x^{6}+2 x^{8}+x^{10}+x^{12}\right) \\
& +a^{35}\left(-x^{9}-x^{11}-x^{13}\right) \\
& +a^{36} \cdot x^{14} .
\end{aligned}
$$

Denominator (as mentioned before) is

$$
=1-a x .1-a x^{3} .1-a x^{5} .1-a x^{7} .1-a^{4} \cdot 1-a^{6} .1-a^{8} .1-a^{10} .1-a^{12} .
$$

The method of calculation is as follows: write for a moment

$$
\phi(a, x)=\frac{1-x^{-2}}{1-a x^{7} \cdot 1-a x^{5} \cdot 1-a x^{3} \cdot 1-a x \cdot 1-a x^{-1} \cdot 1-a x^{-3} \cdot 1-a x^{-5} \cdot 1-a x^{-7}} ;
$$

then $\phi(a, x)$, developed in ascending powers of $a$, and rejecting from the result all negative powers of $x$, is

$$
=\frac{Z_{0}+a Z_{1}+\ldots+a^{36} Z_{36}}{1-a x .1-a x^{3} \cdot 1-a x^{5} \cdot 1-a x^{7} \cdot 1-a^{4} \cdot 1-a^{6} \cdot 1-a^{8} \cdot 1-a^{10} \cdot 1-a^{12}},
$$

developed in like manner in ascending powers of $a$; for the determination of the $Z$ 's up to $Z_{18}$ we require only the development of $\phi(a, x)$ up to $a^{18}$; and, assuming that each $Z$ is at most of the degree 14 in $x$, we require the coefficients of the different powers of $a$ in $\phi(a, x)$ only up to $x^{14}$. Assuming then that $\phi(a, x)$ developed in ascending powers of $a$, up to $a^{18}$, rejecting all negative powers of $x$, and all positive powers greater than $x^{14}$, is

we have

$$
=X_{0}+a X_{1}+\ldots+a^{18} X_{18}
$$

$X_{0}+a X_{1}+\ldots+a^{18} X_{18}=\frac{Z_{0}+a Z_{1}+\ldots+a^{18} Z_{18}}{1-a x \cdot 1-a x^{3} \cdot 1-a x^{5} \cdot 1-a x^{7} \cdot 1-a^{4} \cdot 1-a^{6} \cdot 1-a^{8} \cdot 1-a^{10} \cdot 1-a^{12}}$, or say

$$
\begin{aligned}
Z_{0}+a Z_{1}+\ldots+a^{18} Z_{18}=1-a^{4} \cdot 1-a^{6} \cdot 1-a^{8} \cdot 1-a^{10} \cdot 1-a^{12} . \\
1-a x \cdot 1-a x^{3} \cdot 1-a x^{5} \cdot 1-a x^{7} \cdot\left(X_{0}+a X_{1}+\ldots+a^{18} X_{18}\right)
\end{aligned}
$$

viz. developing here the right-hand side as far as $a^{18}$, but in each term rejecting the powers of $x$ above $x^{14}$, the coefficients of the several powers $a^{0}, a^{1}, \ldots, a^{18}$ give the 
required values $Z_{0}, Z_{1}, \ldots, Z_{18}$. We require, therefore, only to know the values of these functions $X_{0}, X_{1}, \ldots, X_{18}$.

To make a break in the calculation, it is convenient to write

$$
1-a x .1-a x^{3} .1-a x^{5} \cdot 1-a x^{7}\left(X_{0}+a X_{1}+\ldots+a^{18} X_{18}\right)=Y_{0}+a Y_{1}+\ldots+a^{18} Y_{18} ;
$$

putting then

where (up to $\left.x^{14}\right)$

$$
1-a x .1-a x^{3} \cdot 1-a x^{5} .1-a x^{7}=1-a p+a^{2} q-a^{3} r
$$

we have

$$
\begin{aligned}
& p=x+x^{3}+x^{5}+x^{7}, \\
& q=x^{4}+x^{6}+2 x^{8}+x^{10}+x^{12}, \\
& r=x^{9}+x^{11}+x^{13},
\end{aligned}
$$

$$
Y_{0}+a Y_{1}+a^{2} Y_{2}+\ldots+a^{18} Y_{18}=\left(1-a p+a^{2} q-a^{3} r\right)\left(X_{0}+a X_{1}+a^{2} X_{2}+\ldots+a^{18} X_{18}\right) .
$$

The values of $Y_{0}, Y_{1}, \ldots, Y_{18}$ then are

$$
\begin{aligned}
& =\begin{array}{lllll}
Y_{0} & Y_{1} & Y_{2} & Y_{3} \ldots & Y_{18} \\
\hline X_{0} & X_{1} & X_{2} & X_{3} & X_{18}
\end{array} \\
& \begin{array}{llll}
-p X_{0} & -p X_{1} & -p X_{2} & -p X_{17}
\end{array} \\
& +q X_{0}+q X_{1} \quad+q X_{16} \\
& \begin{array}{ll}
-r X_{0} & -r X_{15}
\end{array}
\end{aligned}
$$

the values being taken to $x^{14}$ only; and we then have

$$
Z_{0}+a Z_{1}+a^{2} Z_{2}+\ldots+a^{18} Z_{18}=1-a^{4} \cdot 1-a^{6} \cdot 1-a^{8} \cdot 1-a^{10} \cdot 1-a^{12}\left(Y_{0}+a Y_{1}+\ldots+a^{18} Y_{13}\right) ;
$$

viz. the values of $Z_{0}, Z_{1}, \ldots, Z_{18}$ are

$$
\begin{array}{rlrrrrrrrr}
Z_{0} & Z_{1} & Z_{2} & Z_{3} & Z_{4} & Z_{5} & Z_{6} & Z_{7} & Z_{8} & Z_{9} \\
\hline Y_{0} & Y_{1} & Y_{2} & Y_{3} & Y_{4} & Y_{5} & Y_{6} & Y_{7} & Y_{8} & Y_{9} \\
& & & & -Y_{0} & -Y_{1} & -Y_{2} & -Y_{3} & -Y_{4} & -Y_{5} \\
& & & & & & -Y_{0} & -Y_{1} & -Y_{2} & -Y_{3} \\
& & & & & & & & -Y_{0} & -Y_{1} \\
Z_{10} & Z_{11} & Z_{12} & Z_{13} & Z_{14} & Z_{15} & Z_{16} & Z_{17} & Z_{18} \\
\hline Y_{10} & Y_{11} & Y_{12} & Y_{13} & Y_{14} & Y_{15} & Y_{16} & Y_{17} & Y_{18} \\
-Y_{6} & -Y_{7} & -Y_{8} & -Y_{9} & -Y_{10} & -Y_{11} & -Y_{12} & -Y_{13} & -Y_{14} \\
-Y_{4} & -Y_{5} & -Y_{6} & -Y_{7} & -Y_{8} & -Y_{9} & -Y_{10} & -Y_{11} & -Y_{12} \\
-Y_{2} & -Y_{3} & -Y_{4} & -Y_{5} & -Y_{6} & -Y_{7} & -Y_{8} & -Y_{9} & -Y_{10} \\
& & & & +2 Y_{0} & +2 Y_{1} & +2 Y_{2} & +2 Y_{2} & +2 Y_{4}
\end{array}
$$

The rule of symmetry, before referred to, is that the coefficient $Z_{36-p}$ of $a^{36-p}$ is obtained from the coefficient $Z_{p}$ of $a^{p}$ by changing each power $x^{q}$ into $x^{14-q}$, the coefficients being unaltered; in particular $Z_{18}$, the coefficient of $a^{18}$, must remain 
unaltered when each power $x^{q}$ is changed into $x^{14-q}$; and the verification thus obtained of the value

$$
Z_{18}=2+6 x^{2}+x^{4}+2 x^{6}+2 x^{8}+x^{10}+6 x^{12}+2 x^{14}
$$

is in fact almost a complete verification of the whole work. Some other verifications, which present themselves in the course of the work, will be referred to further on.

We have, therefore, to calculate the coefficients $X_{0}, X_{1}, \ldots, X_{18}$; the function $\phi(a, x)$ regarded as a function of $a$ is at once decomposed into simple fractions; viz. we have

$$
\begin{aligned}
\phi(a, x) & =\frac{1-x^{-2}}{1-a x^{7} \cdot 1-a x^{5} \cdot 1-a x^{3} \cdot 1-a x \cdot 1-a x^{-1} \cdot 1-a x^{-3} \cdot 1-a x^{-5} \cdot 1-a x^{-7}} \\
& =\frac{x^{54}}{1-x^{4} \cdot 1-x^{6} \cdot 1-x^{8} \cdot 1-x^{10} \cdot 1-x^{12} \cdot 1-x^{14}} \frac{1}{1-a x^{7}} \\
& -\frac{x^{40}}{1-x^{2} \cdot 1-x^{4} \cdot 1-x^{6} \cdot 1-x^{8} \cdot 1-x^{10} \cdot 1-x^{12}} \frac{1}{1-a x^{5}} \\
& +\frac{x^{28}}{1-x^{2} \cdot\left(1-x^{4}\right)^{2} \cdot 1-x^{6} \cdot 1-x^{8} \cdot 1-x^{10}} \frac{1}{1-a x^{3}} \\
& -\frac{x^{18}}{1-x^{2} \cdot\left(1-x^{4}\right)^{2} \cdot\left(1-x^{6}\right)^{2} \cdot 1-x^{8}} \frac{1}{1-a x} \\
& +\frac{x^{10}}{1-x^{2} \cdot\left(1-x^{4}\right)^{2} \cdot\left(1-x^{6}\right)^{2} \cdot 1-x^{8}} \frac{1}{1-a x^{-1}} \\
& +\frac{x^{4}}{1-x^{2} \cdot\left(1-x^{4}\right)^{2} \cdot 1-x^{6} \cdot 1-x^{8} \cdot 1-x^{10}} \frac{1}{1-a x^{-3}} \\
& -\frac{1}{1-x^{2} \cdot 1-x^{4} \cdot 1-x^{6} \cdot 1-x^{8} \cdot 1-x^{10} \cdot 1-x^{12}} \frac{1}{1-a x^{-5}} \\
& -\frac{x^{-2}}{1-x^{4} \cdot 1-x^{6} \cdot 1-x^{8} \cdot 1-x^{10} \cdot 1-x^{12} \cdot 1-x^{14}} \frac{1}{1-a x^{-7}} .
\end{aligned}
$$

In order to obtain the expansion of $\phi(a, x)$ in the assumed form of an expansion in ascending powers of $a$, we must of course expand the simple fractions $\frac{1}{1-a x^{7}}$, \&c., in ascending powers of $a$, but it requires a little consideration to see that we must also expand the $x$-coefficients of these simple fractions in ascending powers of $x$. For instance, as regards the term independent of $a$, here developing the several coefficients as far as $x^{18}$, the last five terms give (see post)

$$
\begin{aligned}
& \begin{array}{r}
-x^{18} \\
+x^{10}+x^{12}+3 x^{14}+5 x^{16}+9 x^{18}
\end{array} \\
& -x^{4}-x^{6}-3 x^{8}-4 x^{10}-8 x^{12}-11 x^{14}-18 x^{16}-24 x^{18} \\
& 1+x^{2}+2 x^{4}+3 x^{6}+5 x^{8}+7 x^{10}+11 x^{12}+14 x^{14}+20 x^{16}+26 x^{18}
\end{aligned}
$$

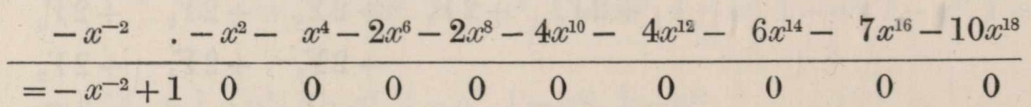

viz. the sum is $=1-x^{-2}$ as it should be*.

* To give the last degree of perfection to the beautiful method of Professor Cayley it would seem desirable that a proof should be given of the principle illustrated by the example in the text, and the nature of the mischief resulting from its neglect clearly pointed out.-EDs. of the A. J. M. 
The expansion is required only as far as $x^{14}$ : the first four terms are therefore to be disregarded, and, writing for shortness

$$
\begin{aligned}
& E=\frac{1}{1-x^{2} \cdot\left(1-x^{4}\right)^{2}\left(1-x^{6}\right)^{2} \cdot 1-x^{8}}, \\
& F=\frac{1}{1-x^{2} \cdot\left(1-x^{4}\right)^{2} \cdot 1-x^{6} \cdot 1-x^{8} \cdot 1-x^{10}}, \\
& G=\frac{1}{1-x^{2} \cdot 1-x^{4} \cdot 1-x^{6} \cdot 1-x^{8} \cdot 1-x^{10} \cdot 1-x^{12}}, \\
& H=\frac{1}{1-x^{4} \cdot 1-x^{6} \cdot 1-x^{8} \cdot 1-x^{10} \cdot 1-x^{12} \cdot 1-x^{14}},
\end{aligned}
$$

we have

$$
\phi(a, x)=\frac{x^{10} E}{1-a x^{-1}}-\frac{x^{4} F^{\prime}}{1-a x^{-3}}+\frac{G}{1-a x^{-5}}-\frac{x^{-2} H}{1-a x^{-7}},
$$

which is

$$
\begin{gathered}
=x^{10} E\left(1+a x^{-1}+a^{2} x^{-2}+\ldots\right) \\
-x^{4} F\left(1+a x^{-3}+a^{2} x^{-6}+\ldots\right) \\
+\quad G\left(1+a x^{-5}+a^{2} x^{-10}+\ldots\right) \\
-x^{-2} H\left(1+a x^{-7}+a^{2} x^{-14}+\ldots\right),
\end{gathered}
$$

where the several series are to be continued up to $a^{18}$, and, after substituting for $E, F, G, H$ their expansions in ascending powers of $x$, we are to reject negative powers of $x$, and also powers higher than $x^{14}$. The functions $E, F, G, H$ contain each of them only even powers of $x$, and it is easy to see that we require the expansions up to $x^{22}, x^{64}, x^{104}$ and $x^{142}$ respectively. For the sake of a verification, I in fact calculated $E, F$ up to $x^{64}$ and $G, H$ up to $x^{142}$ : viz. we have

$$
\left(1-x^{6}\right) E=\left(1-x^{10}\right) F,
$$

from the coefficients of $E$ we have those of $\left(1-x^{6}\right) E$, and in the process of calculating $F$ we have at the last step but one the coefficients of $\left(1-x^{10}\right) F$, the agreement of the two sets being the verification; similarly,

$$
\left(1-x^{2}\right) G=\left(1-x^{14}\right) H
$$

gives a verification. The process for the calculation of $E$,

$$
=\frac{1}{1-x^{2} \cdot\left(1-x^{4}\right)^{2}\left(1-x^{6}\right)^{2} \cdot 1-x^{8}},
$$


is as follows:

\begin{tabular}{|c|c|c|c|c|c|c|c|c|c|c|c|c|}
\hline \multirow{3}{*}{$\left(1-x^{2}\right)^{-1}$} & \multicolumn{2}{|c|}{ Ind. $x$} & \multirow[b]{2}{*}{4} & \multirow[b]{2}{*}{6} & \multirow[b]{2}{*}{8} & \multirow[b]{2}{*}{10} & \multirow[b]{2}{*}{12} & \multirow[b]{2}{*}{14} & \multirow[b]{2}{*}{16} & \multirow[b]{2}{*}{18} & \multirow[b]{2}{*}{20} & \multirow[b]{2}{*}{22} \\
\hline & 0 & 2 & & & & & & & & & & \\
\hline & 1 & 1 & 1 & 1 & 1 & 1 & 1 & 1 & 1 & 1 & 1 & 1 \\
\hline \multirow{3}{*}{$\left(1-x^{4}\right)^{-1}$} & & & 1 & 1 & 2 & 2 & 3 & 3 & 4 & 4 & 5 & 5 \\
\hline & 1 & 1 & 2 & 2 & 3 & 3 & 4 & 4 & 5 & 5 & 6 & 6 \\
\hline & & & 1 & 1 & 3 & 3 & 6 & 6 & 10 & 10 & 15 & 15 \\
\hline \multirow{2}{*}{$\left(1-x^{4}\right)^{-1}$} & 1 & 1 & 3 & 3 & 6 & 6 & 10 & 10 & 15 & 15 & 21 & 21 \\
\hline & & & & 1 & 1 & 3 & 4 & 7 & 9 & 14 & 17 & 24 \\
\hline \multirow[t]{2}{*}{$\left(1-x^{6}\right)^{-1}$} & 1 & 1 & 3 & 4 & 7 & 9 & 14 & 17 & 24 & 29 & 38 & 45 \\
\hline & & & & 1 & 1 & 3 & 5 & 8 & 12 & 19 & 25 & 36 \\
\hline \multirow[t]{2}{*}{$\left(1-x^{6}\right)^{-1}$} & 1 & 1 & 3 & 5 & 8 & 12 & 19 & 25 & 36 & 48 & 63 & 81 \\
\hline & & & & & 1 & 1 & 3 & 5 & 9 & 13 & 22 & 30 \\
\hline$E=\left(1-x^{8}\right)^{-1}$ & 1 & 1 & 3 & 5 & 9 & 13 & 22 & 30 & 45 & 61 & 85 & 111 \\
\hline
\end{tabular}

the alternate lines giving the developments of the functions

$$
\left(1-x^{2}\right)^{-1},\left(1-x^{2}\right)^{-1}\left(1-x^{4}\right)^{-1},\left(1-x^{2}\right)^{-1}\left(1-x^{4}\right)^{-2}, \ldots,
$$

which are the products of the $x$-functions down to any particular line. And in like manner we have the expansions of the other functions $F, G, H$ respectively. I give first the expansions of $E, F, G, H$; next the calculation of the $X$ 's; then the calculation of the $Y$ 's: and from these the $Z$ 's up to $Z_{18}$, or coefficients of the powers $a^{0}, a^{1}, \ldots, a^{18}$ in the numerator of the N.G.F. are at once found; and the coefficients of the remaining powers $a^{19}, \ldots, a^{36}$ are then deduced, as already mentioned.

Writing in the formula $x=0$, we have, for the numerator of the N.G.F. of the invariants, the expression

$$
1-a^{6}+2 a^{8}-a^{10}+5 a^{12}+2 a^{14}+6 a^{16}+2 a^{18}+5 a^{20}-a^{22}+2 a^{24}-a^{26}+a^{32},
$$

agreeing with a result in my "Second Memoir on Quantics," Phil. Trans., t. CxLvI. (1856), [Number 141, vol. II. in this Collection, p. 266]; this, then, was a known result, and it affords a verification, not only of the terms in $x^{0}$, but also of those in $x^{14}$. Thus, in calculating the foregoing expression of the numerator, we obtain $Z_{4}=\left(2 x^{4}+x^{8}+x^{14}\right)$, viz. the term is

$$
a^{4}\left(2 x^{4}+x^{8}+x^{14}\right)
$$

and we thence have the corresponding term $a^{32}\left(1+x^{6}+2 x^{10}\right)$, which, when $x=0$, becomes $=a^{32}$, a term of the numerator for the invariants: and the term $1 x^{14}$ of $Z_{4}$ 
is thus verified, viz. so soon as, in the calculation, we arrive at this term, we know that it is right, and the calculation up to this point is, to a considerable extent, verified. And similarly, in continuing the calculation, we arrive at other terms which are verified in the like manner.

Expansions of the Functions $E, F, G, H$.

\begin{tabular}{crrrr|rrrrr} 
Ind. $x$ & $E$ & $F$ & $G$ & $H$ & Ind. $x$ & $E$ & $F$ & $G$ & $H$ \\
0 & 1 & 1 & 1 & 1 & 16 & 45 & 36 & 20 & 6 \\
2 & 1 & 1 & 1 & 0 & 18 & 61 & 47 & 26 & 7 \\
4 & 3 & 3 & 2 & 1 & 20 & 85 & 66 & 35 & 10 \\
6 & 5 & 4 & 3 & 1 & 22 & 111 & 84 & 44 & 11 \\
8 & 9 & 8 & 5 & 2 & 24 & & 113 & 58 & 16 \\
10 & 13 & 11 & 7 & 2 & 26 & & 141 & 71 & 17 \\
12 & 22 & 18 & 11 & 4 & 28 & & 183 & 90 & 23 \\
14 & 30 & 24 & 14 & 4 & 30 & & 225 & 110 & 26
\end{tabular}

\begin{tabular}{cccc|ccc|cc} 
Ind. $x$ & $F$ & $G$ & $H$ & Ind. $x$ & $G$ & $H$ & Ind. $x$ & $H$ \\
32 & 284 & 136 & 33 & 70 & 2172 & 419 & 108 & 2265 \\
34 & 344 & 163 & 37 & 72 & 2432 & 472 & 110 & 2426 \\
36 & 425 & 199 & 47 & 74 & 2702 & 515 & 112 & 2623 \\
38 & 508 & 235 & 52 & 76 & 3009 & 576 & 114 & 2807 \\
40 & 617 & 282 & 64 & 78 & 3331 & 629 & 116 & 3026 \\
42 & 729 & 331 & 72 & 80 & 3692 & 699 & 118 & 3232 \\
44 & 872 & 391 & 86 & 82 & 4070 & 760 & 120 & 3479 \\
46 & 1020 & 454 & 96 & 84 & 4494 & 843 & 122 & 3708 \\
48 & 1205 & 532 & 115 & 86 & 4935 & 913 & 124 & 3981 \\
50 & 1397 & 612 & 127 & 88 & 5427 & 1007 & 126 & 4240 \\
52 & 1632 & 709 & 149 & 90 & 5942 & 1091 & 128 & 4541 \\
54 & 1877 & 811 & 166 & 92 & 6510 & 1197 & 130 & 4828 \\
56 & 2172 & 931 & 192 & 94 & 7104 & 1293 & 132 & 5164 \\
58 & 2480 & 1057 & 212 & 96 & 7760 & 1416 & 134 & 5481 \\
60 & 2846 & 1206 & 245 & 98 & 8442 & 1525 & 136 & 5850 \\
62 & 3228 & 1360 & 269 & 100 & 9192 & 1663 & 138 & 6204 \\
64 & 3677 & 1540 & 307 & 102 & 9975 & 1790 & 140 & 6609 \\
66 & & 1729 & 338 & 104 & 10829 & 1945 & 142 & 6998 \\
68 & & 1945 & 382 & 106 & & 2088 & &
\end{tabular}


Calculation of the $X^{\prime}$ 's.

Ind. $x$ even or odd according as suffix $X$ is even or odd.

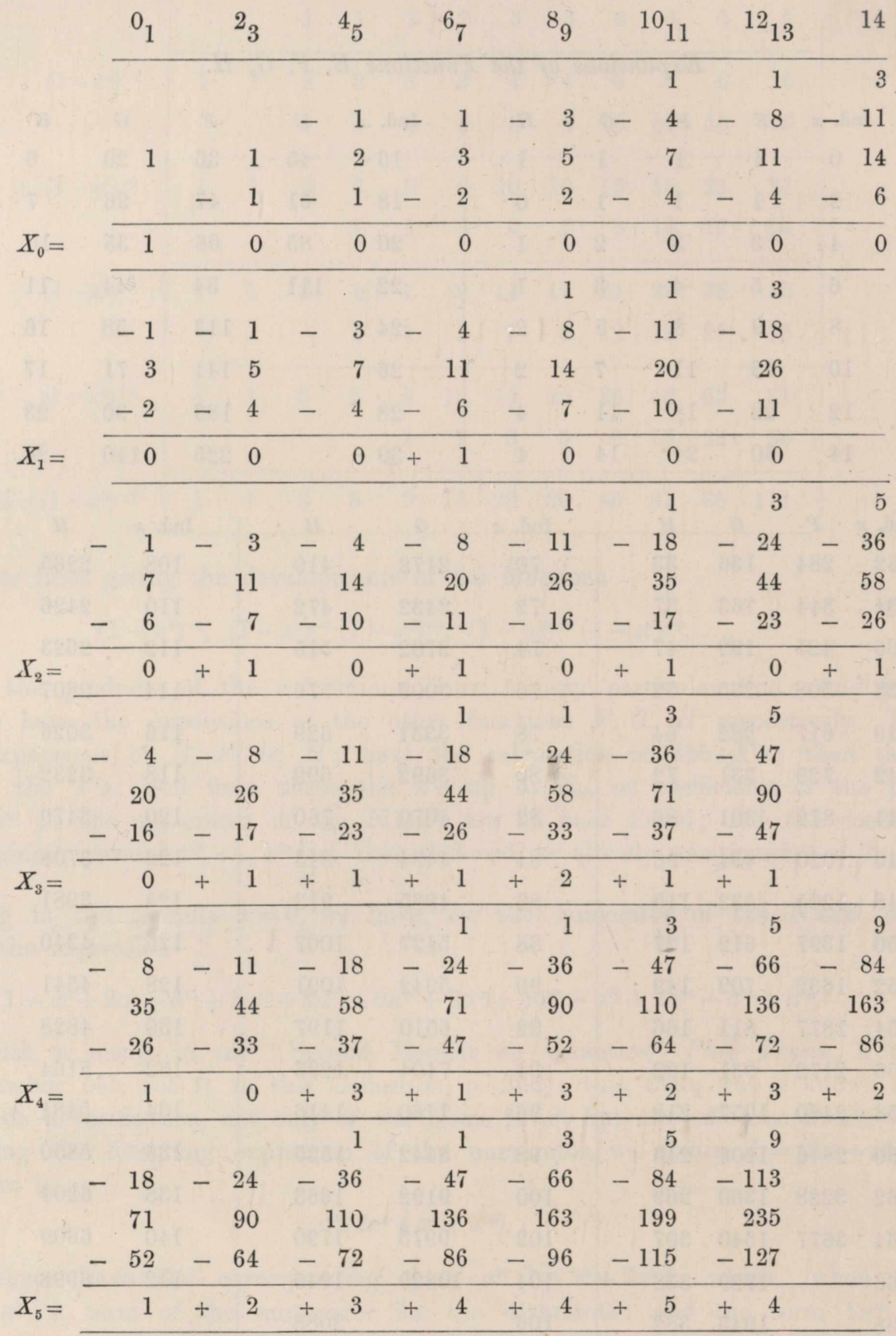




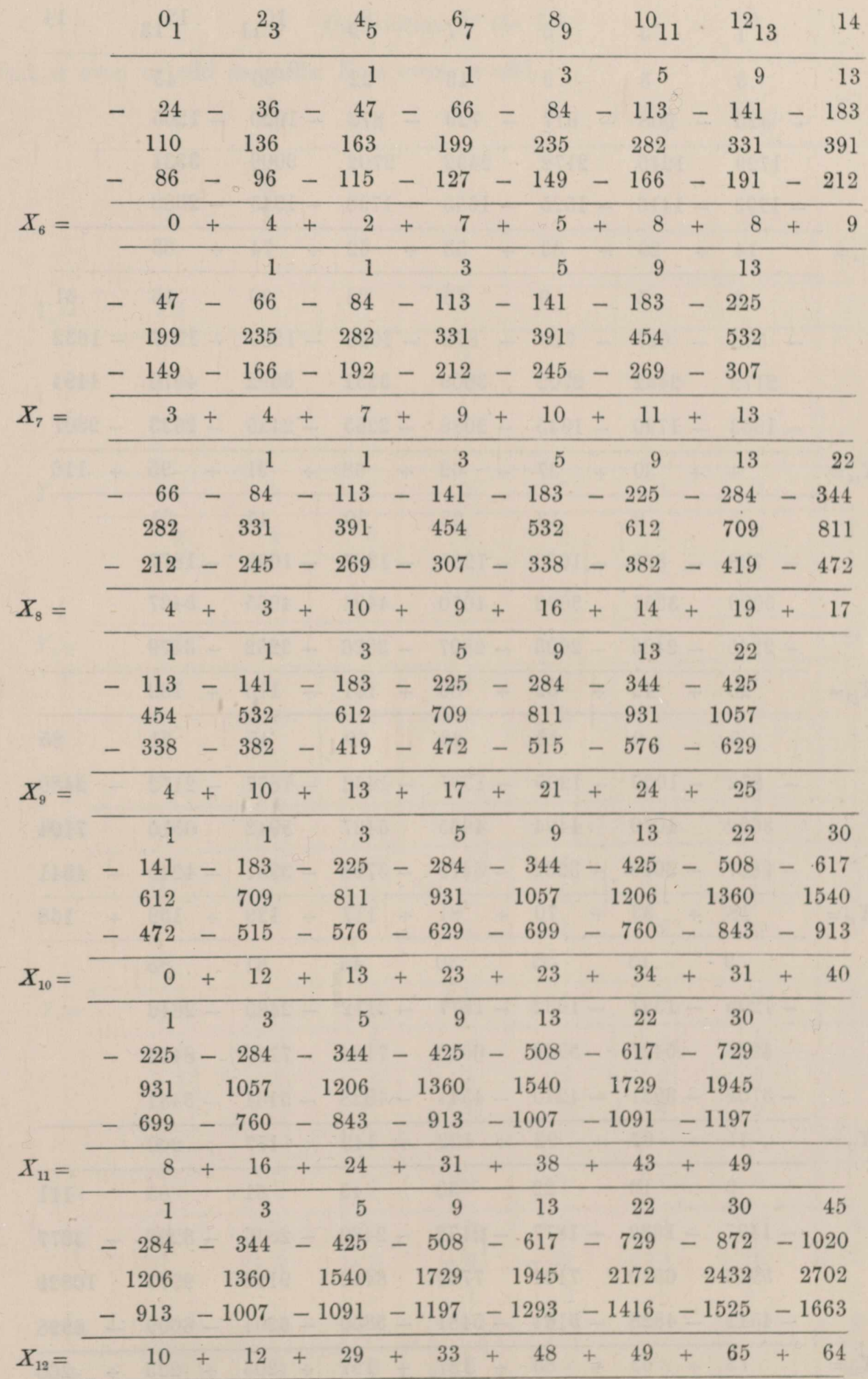

C. $\mathrm{x}$. 
418 CALCULATION OF THE MINIMUM N.G.F. OF THE BINARY SEVENTHIC. [696

$$
\begin{aligned}
& \begin{array}{rrrrrrrr}
{ }_{1} & { }_{3} & { }_{5} & { }_{7} & { }^{8} 9 & { }^{10}{ }_{11} & 12 \\
13 & 14 \\
\hline 3 & 5 & 9 & 13 & 22 & 30 & 45
\end{array} \\
& -425-508-617-729-872-1020-1205 \\
& \begin{array}{lllllll}
1729 & 1945 & 2172 & 2432 & 2702 & 3009 & 3331
\end{array} \\
& -1293-1416-1525-1663-1790-1945-2088 \\
& X_{13}=\frac{14+26+39+53+62+74+83}{}
\end{aligned}
$$

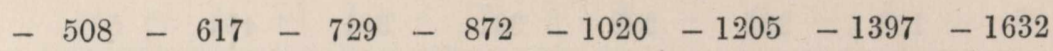

$$
\begin{aligned}
& \begin{array}{llllllll}
2172 & 2432 & 2702 & 3009 & 3331 & 3692 & 4070 & 4494
\end{array} \\
& \begin{array}{llllllll}
-1663 & -1790 & -1945 & -2088 & -2265 & -2426 & -2623 & -2807
\end{array} \\
& X_{14}=\frac{4+30+37+62+68+91+95+116}{5-92613-30-45}
\end{aligned}
$$

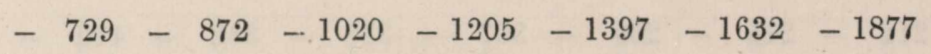

$$
\begin{aligned}
& \begin{array}{lllllll}
3009 & 3331 & 3692 & 4070 & 4494 & 4935 & 5427
\end{array} \\
& \begin{array}{lllllll}
-2265 & -2426 & -2623 & -2807 & -3026 & -3232 & -3479
\end{array}
\end{aligned}
$$

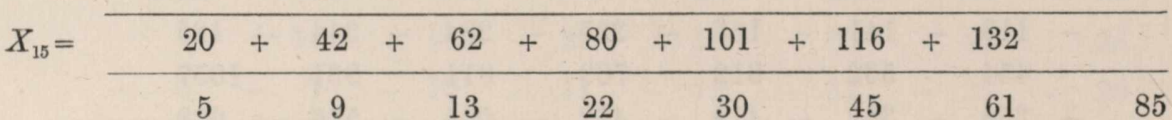

$$
\begin{aligned}
& \text { - } 872-1020-1205-1397-1632-1877-2172-2480 \\
& \begin{array}{llllllll}
3692 & 4070 & 4494 & 4935 & 5427 & 5942 & 6510 & 7104
\end{array} \\
& \begin{array}{llllllll}
-2807 & -3026 & -3232 & -3479 & -3708 & -3981 & -4240 & -
\end{array} \\
& X_{16}=\frac{18+33+70+81+117+129+159+168}{913-22 \quad 30-45-61}
\end{aligned}
$$

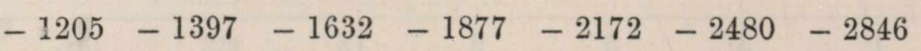

$$
\begin{aligned}
& \begin{array}{lllllll}
4935 & 5427 & 5942 & 6510 & 7104 & 7760 & 8442
\end{array} \\
& -3708-3981-4240-4541-4828-5164-5481
\end{aligned}
$$

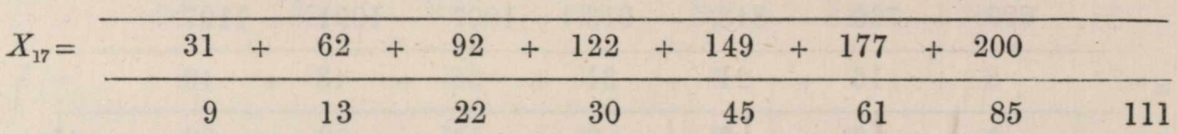

$$
\begin{aligned}
& \begin{array}{llllllll}
-1397 & -1632 & -1877 & -2172 & -2480 & -2846 & -3228 & -
\end{array}-3677 \\
& \begin{array}{llllllll}
5942 & 6510 & 7104 & 7760 & 8442 & 9192 & 9975 & 10829
\end{array} \\
& \begin{array}{llllllll}
-4541 & -4828 & -5164 & -5481 & -5850 & -6204 & -6609 & -6998
\end{array} \\
& X_{18}=13+63+85+137+157+203+223+265
\end{aligned}
$$


Calculation of the $Y$ 's.

Ind. $x$ even or odd as suffix $X$ is even or odd.

\begin{tabular}{llllllll}
${ }_{1}$ & $2_{3}$ & ${ }_{5}$ & $6_{7}$ & $8_{9}$ & ${ }^{10}{ }_{11}$ & ${ }^{12} 13$ & 14 \\
\hline 1 & & & & & &
\end{tabular}

$$
Y_{0}=\frac{1}{1} \begin{array}{llrr}
1 \\
-1 & -1 & -1 & -1
\end{array}
$$

$Y_{1}=$\begin{tabular}{rrrrrrrr}
-1 & -1 & -1 & 0 & 0 & 0 & 0 & \\
\hline 0 & 1 & 0 & 1 & 0 & 1 & 0 & 1
\end{tabular}

$Y_{2}=$\begin{tabular}{rrrrrrrr} 
& & & & -1 & -1 & -1 & -1 \\
\hline 0 & 1 & 1 & 2 & 1 & 1 & 0 & 0 \\
\hline & 1 & 1 & 1 & 2 & 1 & 1 &
\end{tabular}

$Y_{3}=$\begin{tabular}{llrrrrrr} 
& & & & 1 & 1 & \\
& & & & -1 & -1 & -1 & \\
\hline 1 & 0 & 0 & -1 & -1 & -1 & -1 & \\
\hline & & 3 & 1 & 3 & 2 & 3 & 2 \\
& & & -2 & -3 & -5 & -5 & -5 \\
& & & 1 & 1 & 3 & 2 & 4
\end{tabular}

$Y_{4}=$\begin{tabular}{rrrrrrrrr}
1 & 0 & +2 & 0 & +1 & 0 & 0 & +1 \\
\hline 1 & 2 & 3 & 4 & 4 & 5 & 4 \\
-1 & -1 & -4 & -5 & -7 & -9 & -9 & \\
& & & 1 & 2 & 4 & 6
\end{tabular}


CALCULAtion of the Minimum n.G.F. OF the BINARY SEVEnTHic. [696

\begin{tabular}{|c|c|c|c|c|c|c|c|c|}
\hline & $0_{1}$ & ${ }^{2}{ }_{3}$ & $4_{5}$ & $6_{7}$ & $8_{9}$ & ${ }^{10}{ }_{11}$ & ${ }^{12} 13$ & 14 \\
\hline & 3 & 4 & 7 & 9 & 10 & 11 & 13 & \\
\hline & & -4 & -6 & -13 & -18 & -22 & -27 & \\
\hline & & & 1 & 3 & 7 & 12 & 17 & \\
\hline & & & & & -1 & -1 & -4 & \\
\hline$Y_{7}=$ & 3 & 0 & +2 & -1 & -2 & 0 & -1 & \\
\hline & 4 & 3 & 10 & 9 & 16 & 14 & 19 & 17 \\
\hline & & -3 & -7 & -14 & -23 & -30 & -37 & -43 \\
\hline & & & & 4 & 6 & 17 & 20 & 33 \\
\hline & & & & & & $-\quad 1$ & -3 & -6 \\
\hline$Y_{8}=$ & 4 & 0 & +3 & -1 & -1 & 0 & -1 & +1 \\
\hline & 4 & 10 & 13 & 17 & 21 & 24 & 25 & \\
\hline & -4 & -7 & -17 & -26 & -38 & -49 & -58 & \\
\hline & & & 3 & 7 & 17 & 27 & 40 & \\
\hline & & & & & & $-\quad 4$ & -6 & \\
\hline$Y_{9}=$ & 0 & +3 & -1 & -2 & 0 & $-\quad 2$ & +1 & \\
\hline & & 12 & 13 & 23 & 23 & 34 & 31 & 40 \\
\hline & & -4 & -14 & -27 & -44 & -61 & -75 & -87 \\
\hline & & & 4 & 7 & 21 & 29 & 52 & 61 \\
\hline & & & & 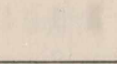 & ff & -3 & -7 & -14 \\
\hline$Y_{10}=$ & 0 & +8 & +3 & +3 & 0 & -1 & +1 & 0 \\
\hline & 8 & 16 & 24 & 31 & 38 & 43 & 49 & \\
\hline & 4 & -12 & -25 & -48 & -71 & -93 & -111 & \\
\hline & & & 4 & 14 & 31 & 54 & & \\
\hline & & & & & -4 & -7 & -17 & \\
\hline$Y_{11}=$ & 8 & +4 & +3 & -3 & -6 & -3 & -1 & \\
\hline & 10 & 12 & 29 & 33 & 48 & 49 & 65 & 64 \\
\hline & & -8 & -24 & -48 & -79 & -109 & -136 & -161 \\
\hline & & & & 12 & 25 & 60 & 84 & 128 \\
\hline & & & & & & $-\quad 4$ & -14 & -27 \\
\hline$Y_{12}=$ & 10 & +4 & +5 & -3 & -6 & -4 & -1 & +4 \\
\hline
\end{tabular}




\begin{tabular}{|c|c|c|c|c|c|c|c|c|}
\hline & ${ }^{0} 1$ & ${ }^{2} 3$ & ${ }^{4} 5$ & $6_{7}$ & $8_{9}$ & ${ }^{10} 11$ & ${ }^{12} 13$ & 14 \\
\hline & 14 & 26 & 39 & 53 & 62 & 74 & 83 & \\
\hline & -10 & -22 & -51 & -84 & -122 & -159 & -195 & \\
\hline & & & 8 & 24 & 56 & 95 & 141 & \\
\hline & & 0 & & & & -12 & -25 & \\
\hline \multirow{5}{*}{$Y_{13}=$} & 4 & +4 & -4 & -7 & -4 & $-\quad 2$ & +4 & \\
\hline & 4 & 30 & 37 & 62 & 68 & 91 & 95 & 116 \\
\hline & & -14 & -40 & -79 & -132 & -180 & -228 & -272 \\
\hline & & & 10 & 22 & 61 & 96 & 161 & 204 \\
\hline & & & & & & -8 & -24 & -48 \\
\hline \multirow[t]{5}{*}{$Y_{14}=$} & 4 & +16 & +7 & +5 & -3 & -1 & +4 & 0 \\
\hline & 20 & 42 & 62 & 80 & 101 & 116 & 132 & \\
\hline & -4 & -34 & -71 & -133 & -197 & -258 & -316 & \\
\hline & & & 14 & 40 & 93 & 158 & 233 & \\
\hline & & & & & -10 & -22 & -51 & \\
\hline \multirow[t]{5}{*}{$Y_{15}=$} & 16 & +8 & +5 & -13 & -13 & -6 & -2 & \\
\hline & 18 & 33 & 70 & 81 & 117 & 129 & 159 & 168 \\
\hline & & -20 & -62 & -124 & -204 & -285 & -359 & -429 \\
\hline & & i & 4 & 34 & 75 & 163 & 238 & 350 \\
\hline & & & & & & -14 & -40 & -79 \\
\hline \multirow[t]{5}{*}{$Y_{16}=$} & 18 & +13 & +12 & -9 & -12 & -7 & -2 & +10 \\
\hline & 31 & 62 & 92 & 122 & 149 & 177 & 200 & \\
\hline & -18 & -51 & -121 & -202 & -301 & -397 & -486 & \\
\hline & & & 20 & 62 & 144 & 246 & 367 & \\
\hline & & & & & -4 & -34 & -71 & \\
\hline \multirow[t]{5}{*}{$Y_{17}=$} & 13 & +11 & $-\quad 9$ & -18 & -12 & -8 & +10 & \\
\hline & 13 & 63 & 85 & 137 & 157 & 203 & 223 & 265 \\
\hline & & -31 & -93 & -185 & -307 & -425 & -540 & -648 \\
\hline & & & 18 & 51 & 139 & 235 & 389 & 511 \\
\hline & & & & & & -20 & -62 & -124 \\
\hline$Y_{18}=$ & 13 & +32 & +10 & $+\quad 3$ & -11 & -7 & +10 & +4 \\
\hline
\end{tabular}

Cambridge, December 7th, 1878. 